\title{
REMARKS ON THE CONTROLLABILITY OF NONLINEAR PERTURBATIONS OF VOLTERRA INTEGRODIFFERENTIAL SYSTEMS
}

\author{
K. BALACHANDRAN and P. BALASUBRAMANIAM \\ Bharathiar University \\ Department of Mathematics \\ Coimbatore- 641 046, Tamil Nadu, India
}

(Received August, 1994; Revised March, 1995)

\begin{abstract}
Sufficient conditions for the complete controllability of nonlinear perturbations of Volterra integrodifferential systems with implicit derivative are established. The results generalize the results of Dauer and Balachandran [9] and are obtained through the notions of condensing map and measure of noncompactness of a set.
\end{abstract}

Key words: Controllability, Integrodifferential Systems, Perturbations, Fixed Point Technique.

AMS (MOS) subject classifications:93B05.

\section{Introduction}

The controllability of perturbed nonlinear systems has been studied by several authors [2-4, 7$9]$ with the help of fixed point theorems. Dacka [6] introduced a new method of analysis to study the controllability of nonlinear systems with implicit derivative based on the measure of noncompactness of a set and Darbo's fixed point theorem. This method has been extended to a larger class of perturbed systems by Balachandran [2,3]. Anichini et al. [1] studied the problem through the notions of condensing map and measure of noncompactness of a set. They used the fixed point theorem due to Sadovskii [11]. In this note, we shall study the controllability of nonlinear perturbations of Volterra integrodifferential systems with implicit derivative by suitably adopting the technique of Anichini et al. [1]. The results generalize the results of Dauer and Balachandran [9].

\section{Preliminaries}

We first summarize some facts concerning condensing maps; for definitions and results about the measure of noncompactness and related topics, the reader can refer to the paper of Dacka [6]. Let $X$ be a subset of a Banach space. An operator $T: X \rightarrow X$ is called condensing if, for any bounded subset $E$ in $X$ with $\mu(E) \neq 0$, we have $\mu(T(E))<\mu(E)$, where $\mu(E)$ denotes the measure of noncompactness of the set $E$ as defined in [11].

We observe that, as a consequence of the properties of $\mu$, if an operator $T$ is the sum of a compact and a condensing operator, then $T$ itself is a condensing operator. Further, if the operator 
$P: X \rightarrow X$ satisfies the condition $|P x-P y| \leq k|x-y|$ for $x, y \in X$, with $0 \leq k<1$, then the operator $P$ has a fixed point property. However, the condition $|P x-P y|<|x-y|$ for $x, y \in X$ is insufficient to ensure that $P$ is a condensing map or that $P$ will admit a fixed point (Browder [5]). The fixed point property holds in the condensing case (Sadovskii [11]).

Let $C_{n}(J)$ denote the space of continuous $R^{n}$ valued functions on the interval $J$. For $x \in C_{n}(J)$ and $h>0$, let

$$
\theta(x, h)=\sup \{|x(t)-x(s)| ; t, s \in J \text { with }|t-s| \leq h\}
$$

and write $\theta(E, h)=\sup _{x \in E} \theta(x, h)$, so that $\theta(E, \cdot)$ is the modulus of continuity of a bounded set $E$. Set $\theta_{0}(E)=\lim _{h \rightarrow 0} \theta\left(\begin{array}{rl}x \in E \\ (E, h\end{array}\right.$. Assume that $\Omega$ is the set of functions $\omega: R^{+} \rightarrow R^{+}$that are right continuous and nondecreasing such that $\omega(r)<r$, for $r>0$. Let $J=\left[t_{0}, t_{1}\right]$.

Lemma 1: [11] Let $X \subset C_{n}(J)$ and let $\beta$ and $\gamma$ be functions defined on $\left[0, t_{1}-t_{0}\right]$ such that $\lim _{s \rightarrow 0} \beta(s)=\lim _{s \rightarrow 0} \gamma(s)=0$. If a transformation $T: X \rightarrow C_{n}(J)$ maps bounded sets into bounded sets such that

$$
\theta(T(x), h)<\omega\left(\theta(x, \beta(h))+\gamma(h) \text { for all } h_{\iota} \in\left[0, t_{1}-t_{0}\right]\right.
$$

and $x \in X$ with $\omega \in \Omega$, then $T$ is a condensing mapping.

Lemma 2: $[1,11]$ Let $X \subset C_{n}\left(\left[t_{0}, t_{1}\right]\right)$, let $I=[0,1]$, and let $S \subset X$ be a bounded closed convex set. Let $H: I \times S \rightarrow X$ be a continuous operator such that, for any $\alpha \in I$, the map $H(\alpha, \cdot)$ : $S \rightarrow X$ is condensing. If $x \neq H(\alpha, x)$ for any $\alpha \in I$ and any $x \in \partial S$ (the boundary of $S$ ), then $H(1, \cdot)$ has a fixed point.

Finally, it is possible to show that for any bounded and equicontinuous set $E$ in $C_{n}^{1}(J)$, the following relations holds:

$$
\mu_{C_{n}^{1}}(E) \equiv \mu_{1}(E)=\mu(D E) \equiv \mu_{C_{n}}(D E)
$$

where $D E=\{\dot{x} ; x \in E\}$.

\section{Main Results}

Consider the nonlinear perturbations of the Volterra integrodifferential system of the form

$$
\begin{aligned}
& \dot{x}(t)=g(t, x)+\int_{t_{0}}^{t} h(t, s, x(s)) d s+B(t, x(t)) u(t) \\
& +f(t, x(t), \dot{x}(t),(S x)(t), u(t)), \ldots, \quad t \in J=\left[t_{0}, t_{1}\right]
\end{aligned}
$$

$x\left(t_{0}\right)=x_{0}$, where the operator $S$ is defined by

$$
(S x)(t)=\int_{0}^{t} k(t, s, x(s)) d s .
$$

Here, $x(t) \in R^{n}, u(t) \in R^{m}$ and the functions $g, h, f, B$ and $k$ satisfy the following hypotheses:

i) $\quad g: J \times R^{n} \rightarrow R^{n}$ is continuous and continuously differentiable with respect to $x$.

ii) $\quad h: J \times J \times R^{n} \rightarrow R^{n}$ is continuous and continuously differentiable with respect to $x$.

iii) $B(t, x(t))$ is a continuous family of matrices on $J \times R^{n}$. 
iv) $\quad f: J \times R^{n} \times R^{n} \times R^{n} \times R^{m} \rightarrow R^{n}$ is continuous.

v) $\quad k: J \times J \times R^{n} \rightarrow R^{n}$ is continuous.

Let $x\left(t, t_{0}, x_{0}\right)$ be the unique solution of the equation

existing on some interval $J$.

$$
\dot{x}(t)=g(t, x)+\int_{t_{0}}^{t} h(t, s, x(s)) d s
$$

Define

$$
G\left(t, t_{0}, x_{0}\right)=g_{x}\left(t, x\left(t, t_{0}, x_{0}\right)\right)
$$

and

$$
H\left(t, s, t_{0}, x_{0}\right)=h_{x}\left(t, s, x\left(s, t_{0}, x_{0}\right)\right)
$$

Then $X\left(t, t_{0}, x_{0}\right)=\frac{\partial}{\partial x_{0}} x\left(t, t_{0}, x_{0}\right)$ exist and is the solution of

such that $X\left(t_{0}, t_{0}, x_{0}\right)=I$.

$$
\dot{y}(t)=G\left(t, t_{0}, x_{0}\right) y(t)+\int_{t_{0}}^{t} H\left(t, s ; t_{0}, x_{0}\right) y(s) d s
$$

Then the solution of the equation (1) is given by [10]

$$
\begin{gathered}
x(t)=x\left(t, t_{0}, x_{0}\right)+\int_{t_{0}}^{t} X(t, s, x(s))[B(s, x) u(s)+f(s, x(s), \dot{x}(s),(S x)(s), u(s))] d s \\
+\int_{t_{0}}^{t} \int_{s}^{t}[X(t, \tau, x(\tau))-R(t, \tau ; s, x(s))] h(\tau, s, x(s)) d \tau d s
\end{gathered}
$$

where $R\left(t, s ; t_{0}, x_{0}\right)$ is the solution of the equation

$$
\frac{\partial R}{\partial s}\left(t, s ; t_{0}, x_{0}\right)+R\left(t, s ; t_{0}, x_{0}\right) G\left(s, t_{0}, x_{0}\right)+\int_{s}^{t} R\left(t, \tau ; t_{0}, x_{0}\right) H\left(\tau, s ; t_{0}, x_{0}\right) d \tau=0
$$

such that $R\left(t, t ; t_{0}, x_{0}\right)=I$ on the interval $t_{0} \leq s \leq t$ and

$$
R\left(t, t_{0} ; t_{0}, x_{0}\right)=X\left(t, t_{0}, x_{0}\right)
$$

We say the system (1) is completely controllable on $J$ if, for any $x_{0}, x_{1} \in R^{n}$, there exists a continuous control function $u(t)$ defined on $J$ such that the solution of (1) satisfies $x\left(t_{1}\right)=x_{1}$. Define the matrix $W$ by

$$
W\left(t, t_{0}, x\right)=\int_{t_{0}}^{t} X(t, s, x(s)) B(s, x(s)) B^{*}(s, x(s)) X^{*}(t, s, x(s)) d s,
$$

where the star denotes the matrix transpose. Further define

$$
q\left(t, t_{0}, x\right)=\int_{t_{0}}^{t} \int_{s}^{t}[X(t, \tau, x(\tau))-R(t, \tau ; s, x(s))] h(\tau, s, x(s)) d \tau d s .
$$


The main results concerning the controllability of the system (1) is given in the following theorem.

Theorem: Let the system (1) satisfy all the above conditions $(i)$ to $(v)$ and assume the additional conditions

(a) $\quad \limsup _{\sin } \frac{|f(t, x, y, S x, u)|}{|x|}=0$,

(b) there exists a continuous nondecreasing function $\omega: R^{+} \rightarrow R^{+}$, with $\omega(r)<r$, such that

$$
|f(t, x, y, S x, u)-f(t, x, z, S x, u)|<\omega(|y-z|) \text { for all }(t, x, y, S x, u) \in J \times R^{3 n} \times R^{m}
$$

(c) there exists a positive constant $\delta$ such that

$$
\operatorname{det} W\left(t_{0}, t_{1}, x\right) \geq \delta \text { for all } x .
$$

Then the system (1) is completely controllable on $J$.

Proof: Define the nonlinear transformation

$$
T: C_{m}(J) \times C_{n}^{1}(J) \rightarrow C_{m}(J) \times C_{n}^{1}(J)
$$

by

$$
T(u, x)(t)=\left(T_{1}(u, x)(t), T_{2}(u, x)(t)\right)
$$

where the pair of operators $T_{1}$ and $T_{2}$ are defined by

$$
\begin{gathered}
T_{1}(u, x)(t)=B^{*}(t, x) X^{*}\left(t_{1}, t, x\right) W^{-1}\left(t_{1}, t_{0}, x\right)\left[x_{1}-x\left(t_{1}, t_{0}, x_{0}\right)\right. \\
\left.-q\left(t_{1}, t_{0}, x\right)-\int_{t_{0}}^{t_{1}} X\left(t_{1}, s, x(s)\right) f(s, x(s), \dot{x}(s),(S x)(s), u(s)) d s\right] \\
T_{2}(u, x)(t)=x\left(t, t_{0}, x_{0}\right)+q\left(t, t_{0}, x\right)+\int_{t_{0}}^{t} X(t, s, x(s)) B(s, x(s)) T_{1}(u, x)(s) d s \\
+\int_{t_{0}}^{t} X(t, s, x(s)) f\left(s, x(s), \dot{x}(s),(S x)(s), T_{1}(u, x)(s)\right) d s .
\end{gathered}
$$

Since all the functions involved in the definition of the operator $T$ are continuous, $T$ is continuous. Moreover, by direct differentiation with respect to $t$, a fixed point for the operator $T$ gives rise to a control $u$ and a corresponding function $x=x(t)$, solution of the system (1) satisfying $x\left(t_{0}\right)=x_{0}, x\left(t_{1}\right)=x_{1}$. Let

$$
\begin{aligned}
& \eta^{0}=\left(u^{0}, x^{0}\right) \in C_{m}(J) \times C_{n}^{1}(J) \\
& \eta=(u, x) \neq 0 \in C_{m}(J) \times C_{n}^{1}(J)
\end{aligned}
$$

and consider the equation

$$
\eta^{0}=\eta-\alpha T(\eta)
$$

where $\alpha \in[0,1]$. This equation can be equivalently written as 


$$
\begin{aligned}
& u=u^{0}+\alpha T_{1}(u, x) \\
& x=x^{0}+\alpha T_{2}(u, x) .
\end{aligned}
$$

From condition (i), for any $\epsilon>0$ there exists $R>0$ such that if $|x|>R$ then $\mid f(t, x, y$, $(S)(x), u)|<\epsilon| x \mid$. Then from (2) we get

$$
\begin{gathered}
|u| \leq\left|u^{0}\right|+|\alpha||B||X|\left|W^{-1}\right|\left[\left|x_{1}\right|+\left|x\left(t_{1}, t_{0}, x_{0}\right)\right|\right. \\
\left.+\left|q\left(t_{1}, t_{0}, x\right)\right|+|X| \epsilon|x| \delta\right] \\
\leq\left|u^{0}\right|+k_{1}+|B||X|^{2}\left|W^{-1}\right| \epsilon \delta|x|
\end{gathered}
$$

where $\delta=t_{1}-t_{0}$ and

$$
k_{1}=|B||X|\left|W^{-1}\right|\left[\left|x_{1}\right|+\left|x\left(t_{1}, t_{0}, x_{0}\right)\right|+\left|q\left(t_{1}, t_{0}, x\right)\right|\right] .
$$

From this inequality and from (3), by applying the Gronwall Lemma, we obtain

$$
\begin{gathered}
|x| \leq\left[\left|x^{0}\right|+\left|x\left(t, t_{0}, x_{0}\right)\right|+\left|T_{1}(u, x)\right||X||B| \delta+\left|q\left(t, t_{0}, x\right)\right|\right] \exp (|X| \epsilon \delta) \\
\leq\left[\left|x^{0}\right|+\left|x\left(t, t_{0}, x_{0}\right)\right|+\left(k_{1}+|B||X|^{2}\left|W^{-1}\right| \epsilon \delta|x|\right)|X||B| \delta\right. \\
\left.+\left|q\left(t, t_{0}, x\right)\right|\right] \exp (|X| \epsilon \delta) .
\end{gathered}
$$

Taking the derivative of (3) with respect to $t$, we obtain

$$
\dot{x}=\frac{d x^{0}}{d t}+\alpha \frac{d}{d t}\left(T_{2}(u, x)(t)\right)
$$

and that results in

$$
\begin{gathered}
|\dot{x}| \leq\left|\dot{x}^{0}\right|+|g(t, x)|+\int_{t_{0}}^{t}|h(t, s, x(s))| d s+|B(t, x(t))|\left|T_{1}(u, x)(t)\right| \\
\quad+|f(t, x(t), \dot{x}(t),(S x)(t), u(t))| \\
\leq\left|\dot{x}^{0}\right|+|g(t, x)|+\int_{t_{0}}^{t}|h(t, s, x(s))| d s+|B|\left[k_{1}+|B||X|^{2}\left|W^{-1}\right| \epsilon \delta|x|\right]+\epsilon|x| \\
=\left|\dot{x}^{0}+k_{2}+\right| x \mid\left[|B|^{2}|X|^{2}\left|W^{-1}\right| \epsilon \delta+\epsilon\right]
\end{gathered}
$$

where $k_{2}=|g(t, x)|+\delta|h(t, s, x(s))|+|B|\left|k_{1}\right|$.

From (4)

$$
|u|-|B||X|^{2}\left|W^{-1}\right| \epsilon \delta|x| \leq\left|u^{0}\right|+k_{1}
$$

and from (5)

$$
|x|\left[\exp (-|X| \epsilon)-|B|^{2}|X|^{3}\left|W^{-1}\right| \epsilon \delta^{2}\right] \leq\left|x^{0}\right|+k_{3}
$$


where $k_{3}=\left|x\left(t, t_{0}, x_{0}\right)\right|+k_{1}|X||B| \delta+\left|q\left(t, t_{0}, x\right)\right|$ and from (6)

$$
|\dot{x}|-|x|\left[|B|^{2}|X|^{2}\left|W^{-1}\right| \epsilon \delta+\epsilon\right] \leq k_{2}+\left|\dot{x}^{0}\right| \text {. }
$$

Taking the sum of all the inequalities (7), (8) and (9), we obtain

$$
\begin{gathered}
|u|-|x|\left\{|B||X|^{2}\left|W^{-1}\right| \epsilon \delta-\exp (-|X| \epsilon \delta)+|B|^{2}|X|^{3}\left|W^{-1}\right| \epsilon \delta^{2}\right. \\
\left.+|B|^{2}|X|^{2}\left|W^{-1}\right| \epsilon \delta+\epsilon\right\}+|\dot{x}| \leq\left|u^{0}\right|+\left|x^{0}\right|+\left|\dot{x}^{0}\right|+k
\end{gathered}
$$

where $k=k_{1}+k_{2}+k_{3}$.

That is,

$$
|u|-\lambda|x|+|\dot{x}| \leq\left|u^{0}\right|+\left|x^{0}\right|+\left|\dot{x}^{0}\right|+k
$$

where $\lambda=|B||X|^{2}\left|W^{-1}\right| \epsilon \delta\{1+|B||X| \delta+|B|\}+\epsilon-\exp (-|X| \epsilon \delta)$.

Then, for suitable positive constants $a, b, c$ we can write

$$
|u|-[\epsilon a-\exp (-\epsilon b)]|x|+|\dot{x}| \leq\left|u^{0}\right|+\left|x^{0}\right|+\left|\dot{x}^{0}\right|+c,
$$

so we divide by $|u|+|x|+|\dot{x}|$ and from the arbitrariness of $\epsilon$, we get the existence of a ball $S$ in $C_{m}(J) \times C_{n}^{1}(J)$ sufficiently large such that

$$
|\eta-\alpha T(\eta)|>0 \text { for } \eta=(u, x) \in \partial S .
$$

We want to show that $T$ is a condensing map. To this aim, we note that $T_{1}: C_{m}(J) \rightarrow C_{m}(J)$ is a compact operator and then, if $E$ is a bounded set, $\mu\left(T_{1}(E)\right)=0$. Then it will be enough to show that $T_{2}$ is a condensing operator. For that, let us consider the modulus of continuity of $D T_{2}(u, x)()$. Now, for $t, s \in J$, we have

$$
\begin{aligned}
& \left|D T_{2}(u, x)(t)-D T_{2}(u, x)(s)\right| \leq|g(t, x(t))-g(s, x(s))|+\mid \int_{t_{0}}^{t} h(t, \tau, x(\tau)) d \tau \\
& \quad-\int_{t_{0}}^{s} h(s, \tau, x(\tau)) d \tau|+| B(t, x(t)) T_{1}(u, x)(t)-B(s, x(s)) T_{1}(u, x)(s) \mid \\
& +\left|f\left(t, x(t), \dot{x}(t),(S x)(t), T_{1}(u, x)(t)\right)-f\left(s, x(s), \dot{x}(s),(S x)(s), T_{1}(u, x)(s)\right)\right|
\end{aligned}
$$

For the first three terms of the right hand side of the inequality, we may given the upper estimate as $\beta_{0}(|t-s|)$ with $\lim _{h \rightarrow 0} \beta_{0}(h)=0$ and it may be chosen independent of the choice of $(u, x)$. For the fourth term, we can given the following estimate:

$$
\begin{aligned}
& \left|f\left(t, x(t), \dot{x}(t),(S x)(t), T_{1}(u, x)(t)\right)-f\left(s, x(s), \dot{x}(s),(S x)(s), T_{1}(u, x)(s)\right)\right| \\
\leq & \left|f\left(t, x(t), \dot{x}(t),(S x)(t), T_{1}(u, x)(t)\right)-f\left(t, x(t), \dot{x}(s),(S x)(t), T_{1}(u, x)(t)\right)\right| \\
+ & \left|f\left(t, x(t), \dot{x}(s),(S x)(t), T_{1}(u, x)(t)\right)-f\left(s, x(s), \dot{x}(s),(S x)(s), T_{1}(u, x)(s)\right)\right| .
\end{aligned}
$$

For the first term we have the upper estimate $\omega(|\dot{x}(t)-\dot{x}(s)|)$ whereas for the second term, 
we may find an estimate

$$
\beta_{1}(|t-s|) \text { with } \lim _{h \rightarrow 0} \beta_{1}(h)=0
$$

Hence

$$
\theta\left(D T_{2}(u, x), h\right) \leq \omega(\theta(D E, h)+\beta(h)
$$

where $\beta=\beta_{0}+\beta_{1}$. Therefore, by Lemma 1 , we get

$$
\theta_{0}\left(D T_{2}(E)\right)<\theta_{0}(D E)
$$

Hence, from

$$
\begin{gathered}
\left.2 \mu_{1}\left(T_{2}(E)\right)=2 \mu\left(D T_{2}(E)\right)=\theta_{0}\left(D T_{2}(E)\right)<\theta_{0}(D E)\right) \\
=2 \mu(D E)=2 \mu_{1}(E)
\end{gathered}
$$

it follows that $\mu_{1}\left(T_{2}(E)\right)<\mu_{1}(E)$. Then the existence of a fixed point of the operator $T$ follows from Lemma 2. In other words, there exists functions $u \in C_{m}(J)$ and $x \in C_{n}^{1}(J)$ such that

$$
T(u, x)=(u, x)
$$

and

$$
u(t)=T_{1}(u, x)(t), \quad x(t)=T_{2}(u, x)(t)
$$

These functions are the required solutions. Further, it is easy to verify that the function $x(\cdot)$ given by the systems (1) satisfies the boundary conditions $x\left(t_{0}\right)=x_{0}$ and $x\left(t_{1}\right)=x_{1}$. Hence, the system (1) is completely controllable.

\section{Acknowledgements}

The authors are grateful to Professor Jewgeni Dshalalow for his kind help. This work is supported by a grant from CSIR, New Delhi.

\section{References}

[1] Anichini, G., Conti, G. and Zecca, P., A note on controllability of certain nonlinear systems, Note Mat., 6 (1986), 99-111.

[2] Balachandran, K., Controllability of a class of perturbed nonlinear systems, Kybernetika, 24 (1988), 61-64.

[3] Balachandran, K. Controllability of perturbed nonlinear systems, IMA J. Math. Control Inform., 6 (1989), 253-258.

[4] Balachandran, K. and Dauer, J.P., Controllability of nonlinear systems via fixed point theorems, J. Optim. Theory Appl., 53 (1987), 345-352.

[5] Browder, F.E., Nonexpansive nonlinear operators in Banach space, Proc. Nat. Acad. Sci., 54 (1965), 1041-1044.

[6] Dacka, C., On the controllability of a class of nonlinear systems, IEEE Trans. Automat. Control, AC-25 (1980), 263-266. 
[7] Dauer, J.P., Nonlinear perturbations of quasilinear systems, J. Math. Anal. Appl., 54 (1976), 717-725.

[8] Dauer, J.P., Controllability of perturbed nonlinear systems, Rend. Acad. Nazion. Linc., 63 (1977), 345-350.

[9] Dauer, J.P. and Balachandran, K., A note on the controllability of nonlinear perturbations of Volterra integrodifferential systems, J. Math. Sys. Estim. Control, 3 (1993), 321-326.

[10] Hu, S., Lakshmikantham, V. and Rama Mohan Rao, M., Nonlinear variation of parameters formula for integrodifferential equations of Volterra type, J. Math. Anal. Appl., 129 (1988), 223-230.

[11] Sadovskii, J.B., Limit compact and condensing operators, Russian Math. Surveys, 27 (1972), 85-155. 


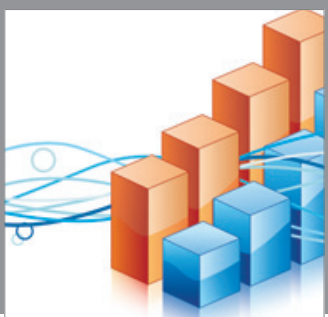

Advances in

Operations Research

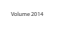

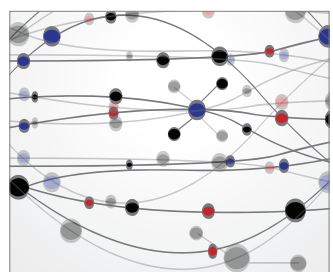

\section{The Scientific} World Journal
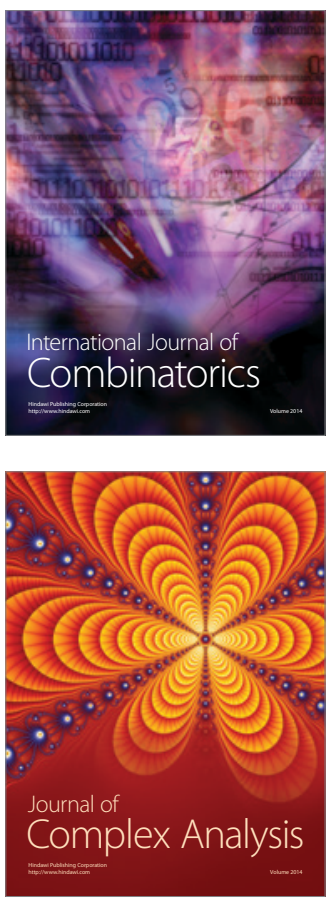

International Journal of

Mathematics and

Mathematical

Sciences
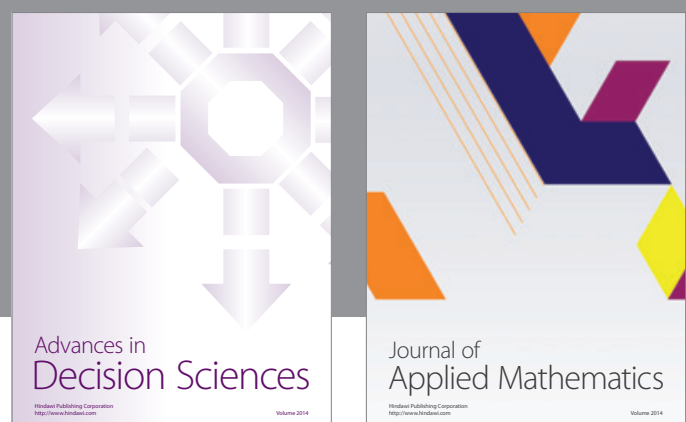

Journal of

Applied Mathematics
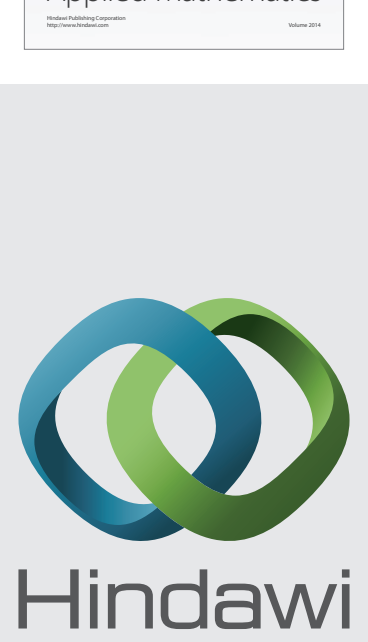

Submit your manuscripts at http://www.hindawi.com
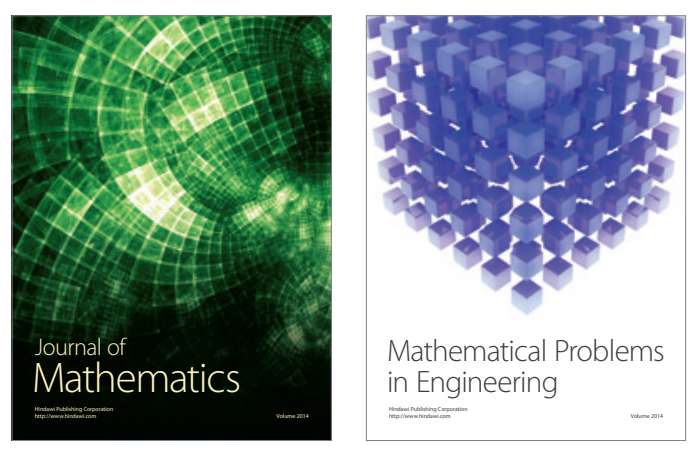

Mathematical Problems in Engineering
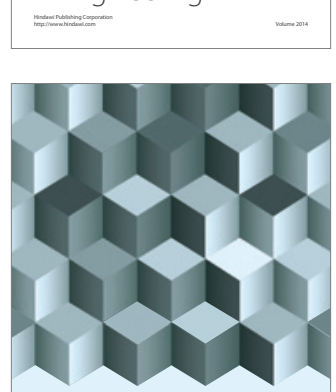

Journal of

Function Spaces
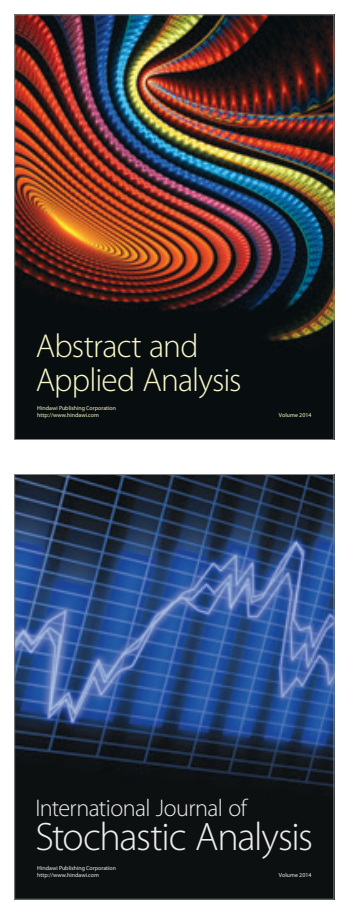

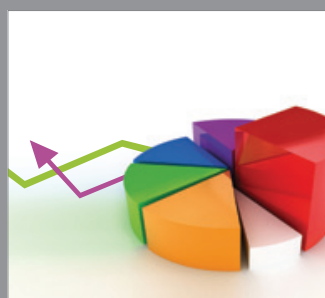

ournal of

Probability and Statistics

Promensencen
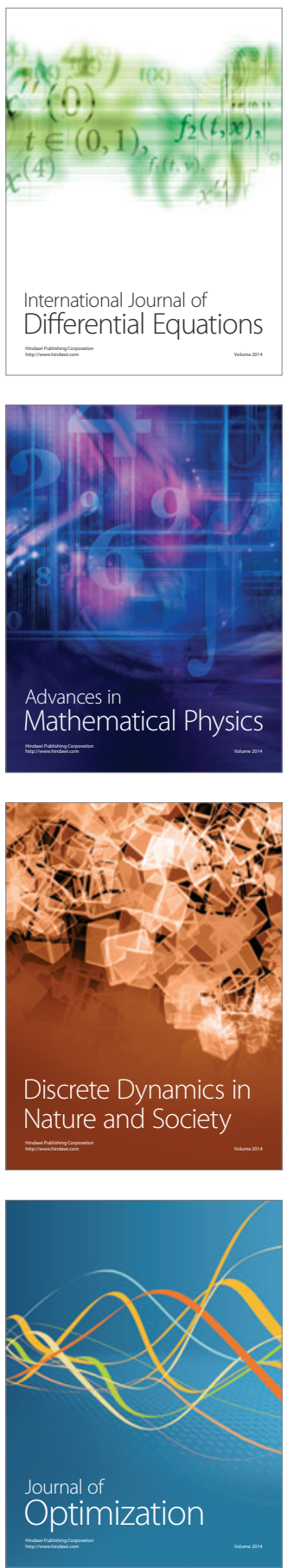